\title{
Literature Survey on Underwater Threat Detection
}

\author{
Taofeek Biobakua, Gino J. Limª, Jaeyoung Choa, \\ Selim Bora ${ }^{b}$, Hamid Parsaei ${ }^{b}$
}

This paper presents a concise review on extant literature in threat detection within the maritime domain with specific emphasis on solution methodologies, sensing technologies, regional affiliations of authors and research contributions related to underwater threat detection. The review attempts to provide an introductory framework on threat detection and surveillance within the maritime domain as opposed to its widely studied terrestrial counterpart. In all, over 200 engineering/science journal papers and conference proceedings were identified. Of these, a total of 136 articles are eventually chosen and reviewed. The analysis of this review suggests that opportunities abound in the research area, especially in Operations Research/ Management Science. Specifically, Sensor placement and deployment for underwater threat detection is identified as a significant sub-research area worth studying.

\section{KEY WORDS}

$\sim$ Literature survey

$\sim$ Underwater threat detection

$\sim$ Operations research/management science

$\sim$ Sensor placement and deployment

a. Department of Industrial Engineering, University of Houston, Houston, TX e-mail:jcho12@uh.edu

b. Texas A\&M University at Qatar, Doha, Qatar

e-mail: selim.bora@qatar.tamu.edu

\section{INTRODUCTION}

World trade is heavily dependent on maritime transportation (Organization for Economic Co-operation and Development, OECD, 2003). This dependence indicates global distribution of economic goods is mostly done via oceans, seas, estuaries, and other maritime waterways. In fact, it is widely accepted that over $90 \%$ of global economic trade utilizes maritime infrastructures and that the maritime sector remains the cheapest medium to transport goods over long distances (The International Maritime Organization, IMO, 2012). Particularly, the US economy is largely dependent on the maritime sector as $95 \%$ of its foreign trade is moved by ship (United States Department of Transportation Maritime Administration, 2007).

In addition, countries have become increasingly dependent on each other for commercial goods, machineries, mineral resources, agricultural produce, etc. Indeed, the new era of global interdependence (IMO, 2012) via maritime industry has come to stay. To ensure international economic trade is not adversely affected, the continued success of the maritime industry needs to be assured. The unique position the maritime industry holds in the world economy (without even addressing its importance in military warfare) accentuates the need to protect its infrastructural resources from threats and disasters. Apart from the scourge of terrorism which the entire global economy is currently experiencing, the age-old threats of piracy, arson attacks and unfortunate accidents are incidences the maritime industry has to contend with.

Similar to the use of terrestrial sensors in the identification and classification of land-based threats and targets, underwater 
sensors are used in the maritime domain for the same purpose. While literature on the use of terrestrial sensors for surveillance is rich and extensive, its underwater counterparts have not been well addressed (Heidemann et al., 2012). In this study, literature related to underwater threat detection is presented irrespective of specific problem domains, solution methodologies or sensing technology. The authors' opinion is that a better understanding of general issues related to underwater threat detection will be an invaluable introduction to the research area, assist in identification of voids in literature and ultimately stimulate future research directions.

For the purpose of this review, literature search is undertaken by examining papers published in year 2000 and onwards with an exception of two closely related papers (published in 1995 and 1999) outside this time frame. Paper classification based on authors' contributions is categorized under Model, Theory and Application developments (Galindo and Batta, 2013; Altay and Green, 2006). Though these contribution categories seem explicit, readers interested in the classification details are referred to these two cited papers. In addition, categories based on authors' regional affiliations, solution methodologies and sensing technologies are included. During the course of this study, the authors identified two review papers (Heidemann et al., 2006; Heidemann et al., 2012) related to underwater techniques. However, neither of these papers considers all available sensing technologies in threat detection.

The remaining sections of this paper is arranged thus: section 2 highlights the scope of the study and search methodology adopted, section 3 presents underlying features identified from articles based on introduced classification schemes and in section 4, the paper is concluded with a discussion on the review's revelations. In addition, a supplementary material (spreadsheet containing a list of all bibliography examined, along with relevant tables and figures) is provided.

\section{SCOPE OF STUDY AND ADOPTED SEARCH METHODOLOGY}

This section highlights and discusses the scope and the search methodology adopted in this study. The scope is limited to threat detection and/or surveillance within a maritime domain. Although several papers related to general and terrestrial threat detection were identified during the course of the literature search, only those specifically related to the marine domain were included. As indicated in section 1, the search period is from year 2000 onwards, with the exception of two papers published in 1995 and 1999. Inclusion of these two papers is due to the inevitable issue of subjectivity when manual scrutiny is involved in any paper review activity. (Altay and Green, 2006), (Natarajarathinam et al., 2009), and (Galindo and Batta, 2013) are papers known to have also acknowledged this issue.
In addition, the chosen articles are limited to published journals and conference proceedings papers. Book chapters, dissertations, technical reviews, etc. are excluded from this research. In addition, the language of publication is solely restricted to the English medium. Limiting the search to only articles related to science and engineering, the total number of articles reviewed is 136, and key search words include: underwater protection", "protection of maritime assets", "protection of maritime resources", and "underwater surveillance.

Consulted database source is divided into two categories: primary and secondary. The former source utilized the "One Search" feature from the University of Houston library systems where unified access is granted to several known databases: SCOPUS, ScienceDirect, ISI Web of Knowledge, etc. The latter source is the online "Google Scholar". In addition, it also serves as the citation source for all bibliography included in the supplementary material. It should be noted that a considerable number of literature related to commercial product developments and reviews are obtained from this latter source. While the authors are aware that not all journal publications have on-line versions, such publications are excluded in this literature search. The justification is that if papers with such an origin related to our review exist; their number will be insignificant, compared to those available online. Moreover, the preferred publication period is from the year 2000 and beyond; thus, it is expected that most relevant papers would be readily available online.

As a basis for literature inclusion, this paper also made use of two distinct screening procedures: primary and secondary screenings. In the primary screening, appraisals of a literature's Title, Abstract and Conclusion sections are carried out. A satisfactory assessment that the article identifies with the problem scope ensures inclusion in the pool of literature; otherwise, the article is excluded. In a situation where doubt is expressed about including or excluding a paper under this screening, a brief perusal of the article's major sections is undertaken. The secondary screening serves as a final filter where a more detailed inspection of the article is executed in order to have a holistic comprehension of the authors' works and contributions. In few instances, papers which were initially included after the Primary screening procedure were ultimately excluded from the review during this latter stage.

\section{UNDERLYING FEATURES IDENTIFIED FROM ARTICLES}

Out of over 200 papers consulted, a total of 136 papers were eventually included in this review paper. It is acknowledged that there could be a possible existence of other papers meeting the scope of study but which have been inadvertently excluded from this review. However, the authors aver that the included papers are a fair representation of bibliography related to the problem area of interest within the time period under review. 
Figure 1 shows the distribution of reviewed papers per journal of publication. The "Others" category include journals with less than two representative journals. As depicted in the figure, a substantial majority (76) of the included papers belong to the IEEE community. All peer-reviewed journals and conference proceedings (including any joint collaboration with other professional associations) affiliated to IEEE are included under this single journal name. Of the main stream OR/MS related journals, only the "Operations Research" journal is represented in this review with a single paper. The other journals with single paper representations can be seen in the supplementary material.

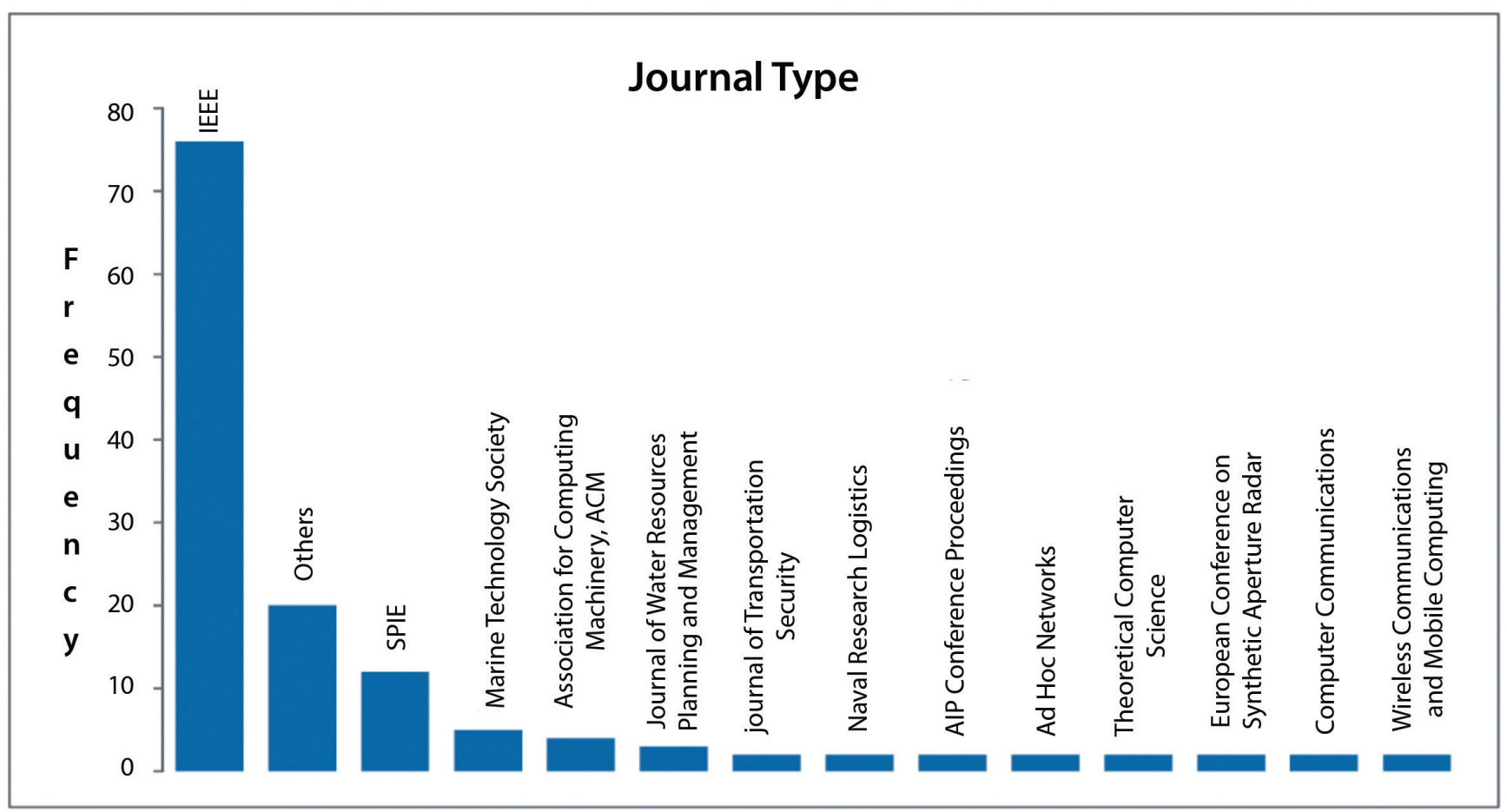

Figure 1.

Distribution of reviewed literature: Journal Type.

\subsection{Authors' Regional Affiliations}

Table 1 and Figure 2 show distributions of the authors' geographical regional affiliations in the reviewed papers. Regional affiliation is indicative of the authors' affiliated institutions (or organizations) and not necessarily their nationalities. Expectedly, the US/Canada region has the most representation. Within the
US/Canada region, the US has $95 \%$ of the reviewed articles and Canada accounts for the rest (see Figure 3). The figure also shows that China and Japan, respectively, occupies $90 \%$ and $10 \%$ of literature from the Asia region. Countries represented within the Europe region are shown in Figure 4, with Italy having the highest visibility at $34 \%$. 
Table 1.

Regional Affiliation: Frequency distribution of reviewed literature.

\section{Region}

US/Canada

Europe

Asia

Australia

International

Total

\section{Frequency}

64

41

10

4

17

136

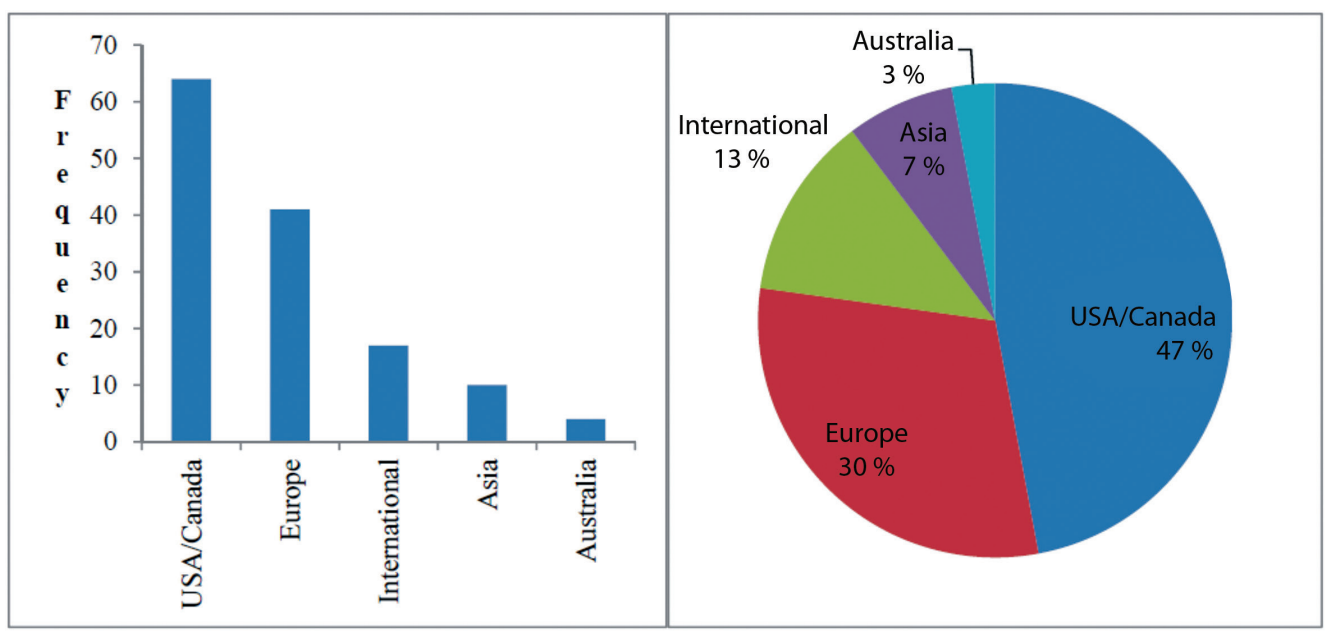

Figure 2.

Regional Affiliations: Distribution of reviewed literature.

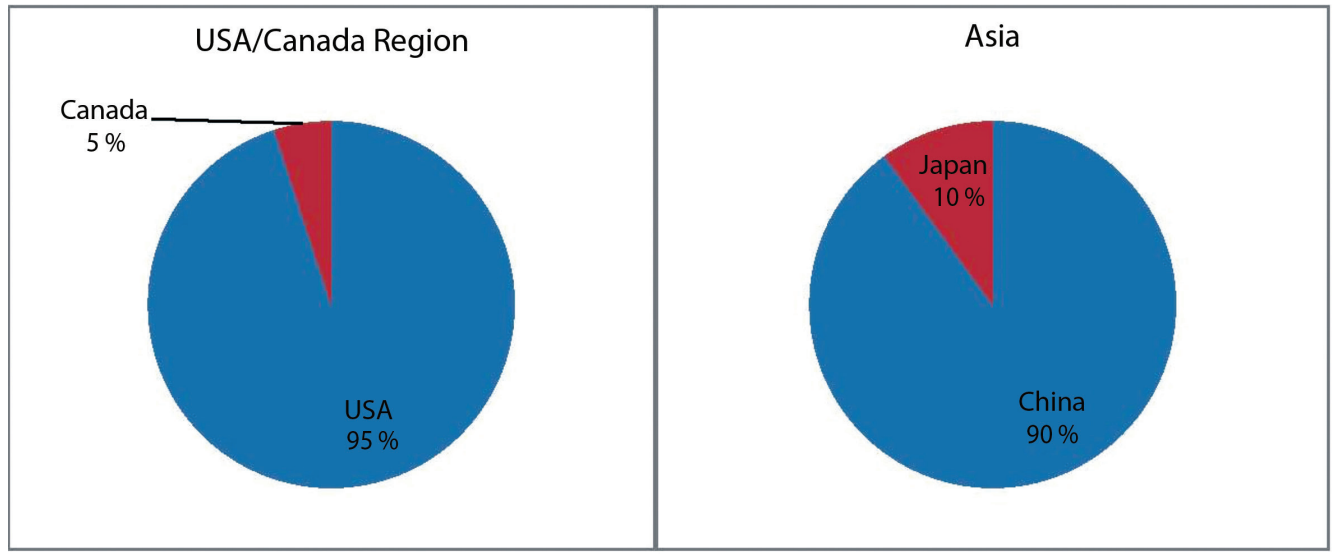

Figure 3.

National Affiliations, USA/Canada and Asia: Distribution of reviewed literature. 


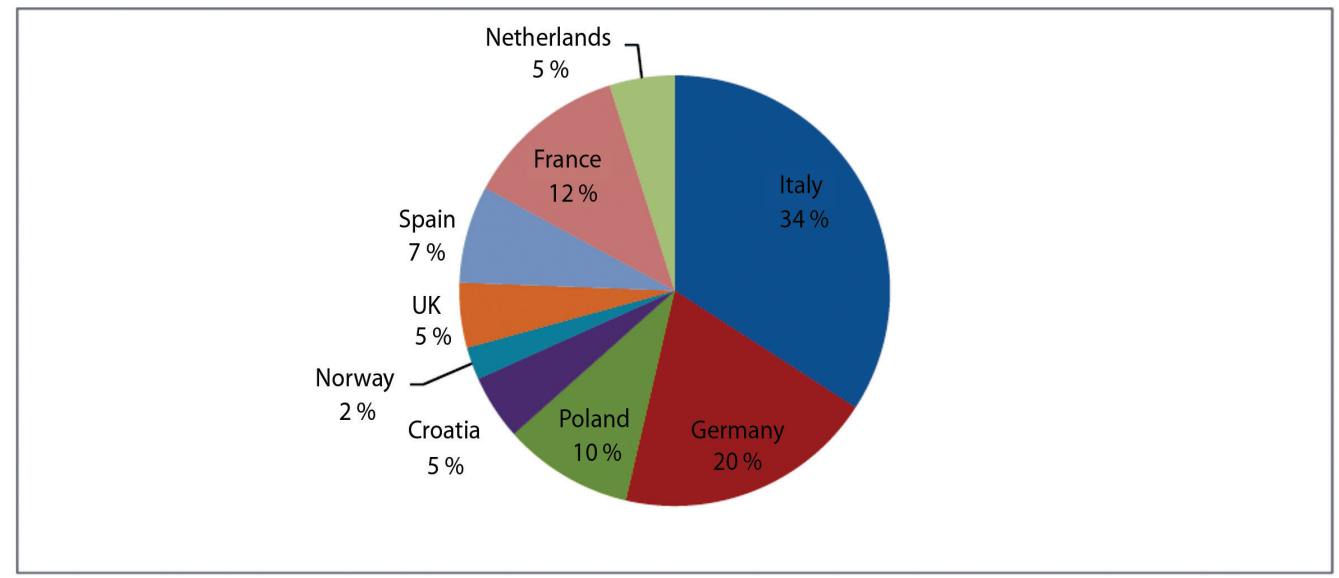

Figure 4.

National Affiliations- Europe: Distribution of reviewed literature.

A large majority of countries from these hitherto highlighted regions are also involved in international collaborations. Apart from these, other countries participating in international partnerships include: Saudi Arabia, Belgium, Mexico, Hong Kong, Portugal, Croatia and Turkey.

It should be noted that the active involvement of the Department of Homeland Security, DHS, and North Atlantic Treaty Organization, NATO, respectively in the US/Canada and Europe regions is a huge contributory factor to the preponderance of literature from these regions. NATO presence and collaborations with research institutions in European countries such as Croatia, Poland, Italy and Norway are worth mentioning in this regard.

\subsection{Solution Approach/Methodology}

The literature survey suggests the use of optimization techniques as the most common methodology in the reviewed literature. MIP (Mixed Integer Programming), ILP (Integer linear programming), Quadratic programming and Stochastic optimization are some of the OR techniques employed by the various authors. In addition, most literature presents the heuristics and algorithms developed to solve large scale problems. Simulation techniques, especially Monte Carlo simulation and numerical simulation are also widely observed in the literature reviewed. As seen in Figure 5, other solution techniques/ methodologies observed include Statistical/Probability Analysis, Game theory, Acoustic analysis, Signal processing analysis, etc. Often times, these methodologies are usually combined with one another. For example, a frequent combination of both Simulation and Optimization techniques is replete in the literature reviewed and this is in consonance with recent publication trends. Hence, the sum total of the reported techniques does not tally to the total number (136) of literature surveyed.

In addition, this review has highlighted papers related to Application Development of surveillance systems as a classification under the Solution Approach/Methodology category. Some of these papers focused on a specific technology/ application/software suite developed to solve some specific maritime surveillance problem(s) in a specific location. Yet, others focused on developing generic solutions to address some surveillance problems. It should also be noted that the specific methodologies adopted in these categories have been appropriately identified. Thus, a paper based on the specific development of a surveillance system (including protocols, hardware, technology, etc.) that introduces a novel methodology (such as statistical analysis or mathematical modeling) is simultaneously classified under "Application Development" as well as the related methodology. 


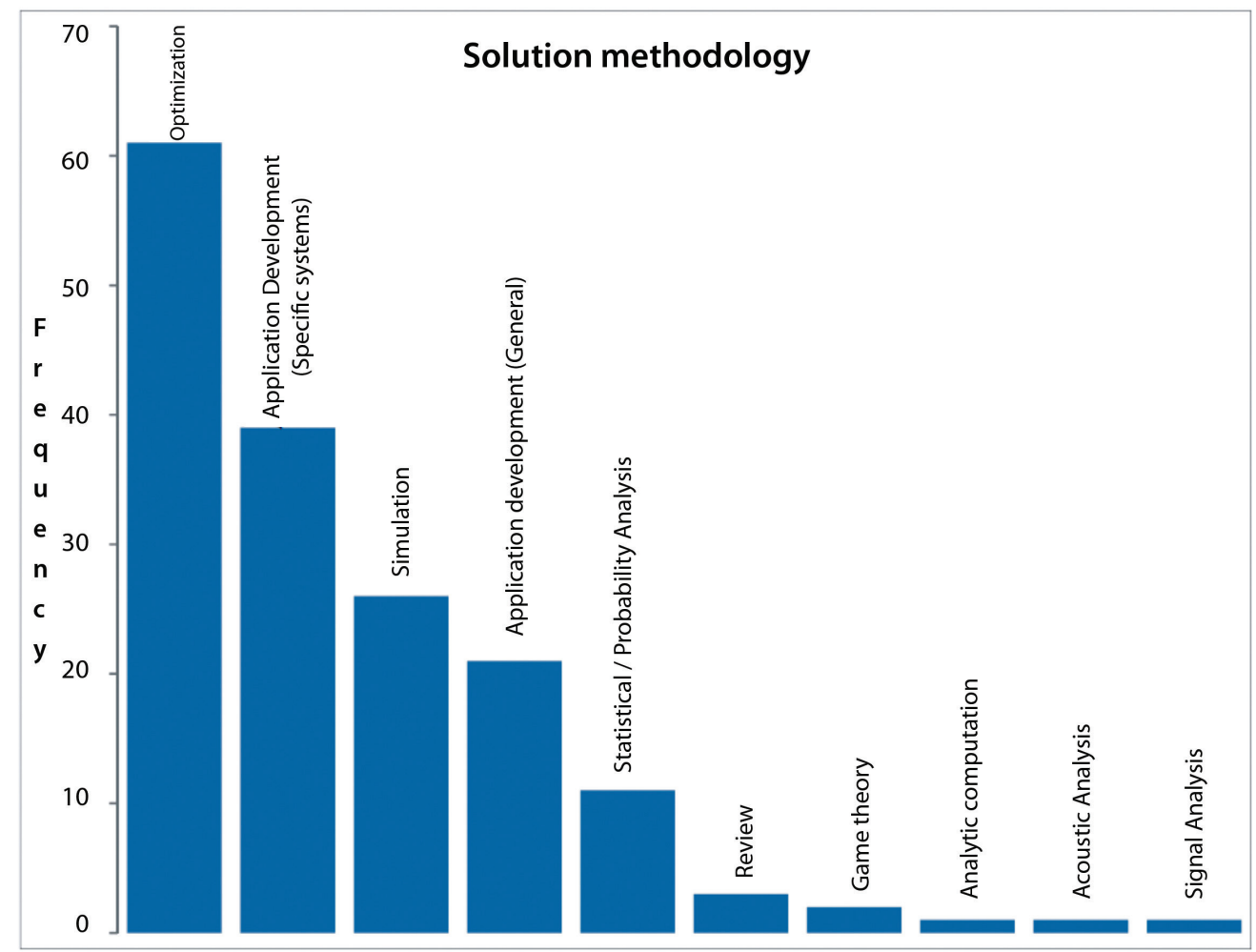

Figure 5.

Methodology: Distribution of reviewed literature.

\subsection{Research Contributions}

Similar to a classification approach adopted in (Altay and Green, 2006) and more recently in Galindo and Batta (2013),
"Theory", "Model", and "Application" developments are identified as the categories under the authors' research contributions.

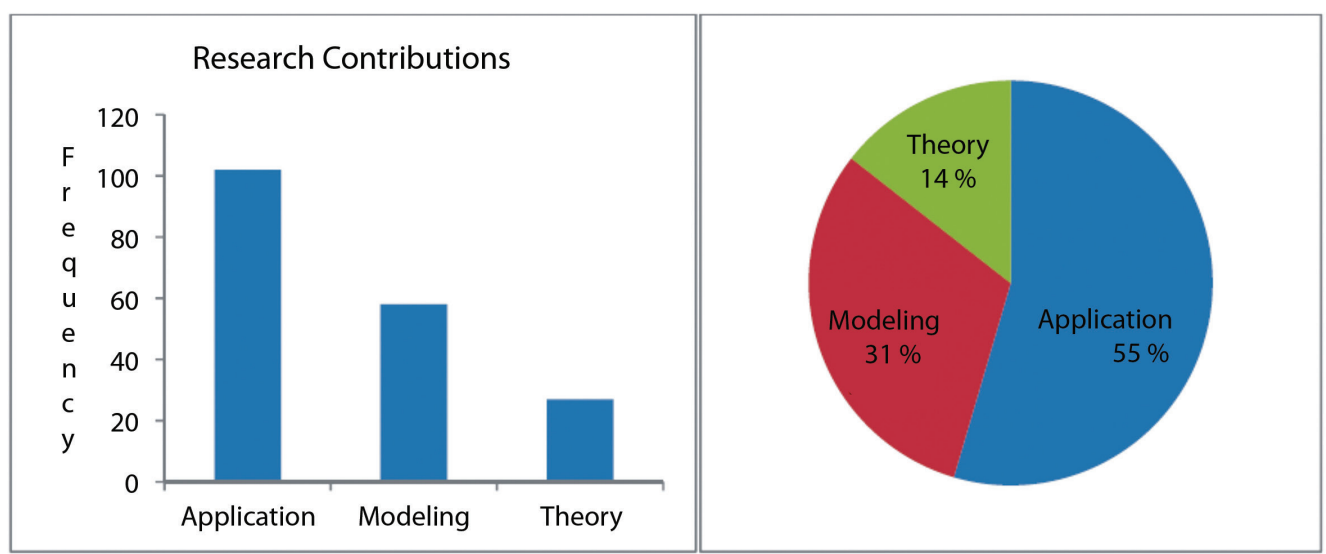

Figure 6.

Research Contributions: Distribution of reviewed literature. 
Like the Solution Approach/Methodology classification, the total number of the literature under the Research contribution category does not tally to the total number (136) of literature

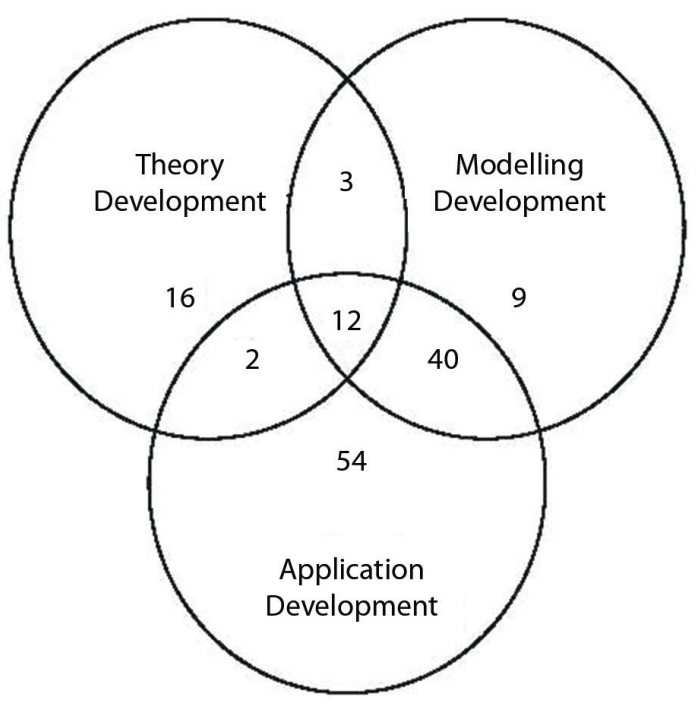

Figure 7.

Research Contributions- Venn diagram: Distribution of reviewed literature. surveyed. Similar reason as in the former category exists for this observation. The Venn diagram in Figure 7 shows the overlap in the classifications under the Research Contributions category.

Of the total number of literature reviewed, only 38 papers (about $28 \%$ ) are related to the Sensor placement problem, SPP (either nodal or network placements), a widely studied problem in Optimization theory. Some of these works surveyed include (Ghafoori and Altiok, 2012; Stolkin and Florescu, 2007; Akkaya and Newell, 2009; Ibrahim et al., 2010; Ngatchou et al., 2006; Molyboha and Zabarankin, 2012), etc. Similar to the terrestrial/ generic sensor placement problem, the SPP is often addressed and solved as a coverage problem, with specific underwater environments taken into consideration. Irrespective of the research contribution, the sensor placement problem for threat detection within the maritime domain is identified as an area that requires researchers' attention.

\subsection{Sensing Technology distributions}

Of all the sensing technologies observed in the surveyed literature 72 (about $73 \%$ ) are acoustic-based. The other sensors observed are as shown in Figure 8. As indicated in the figure, some papers studied more than one type of sensors while other literatures simply stated the use of sensors in their works without specifying the actual types.

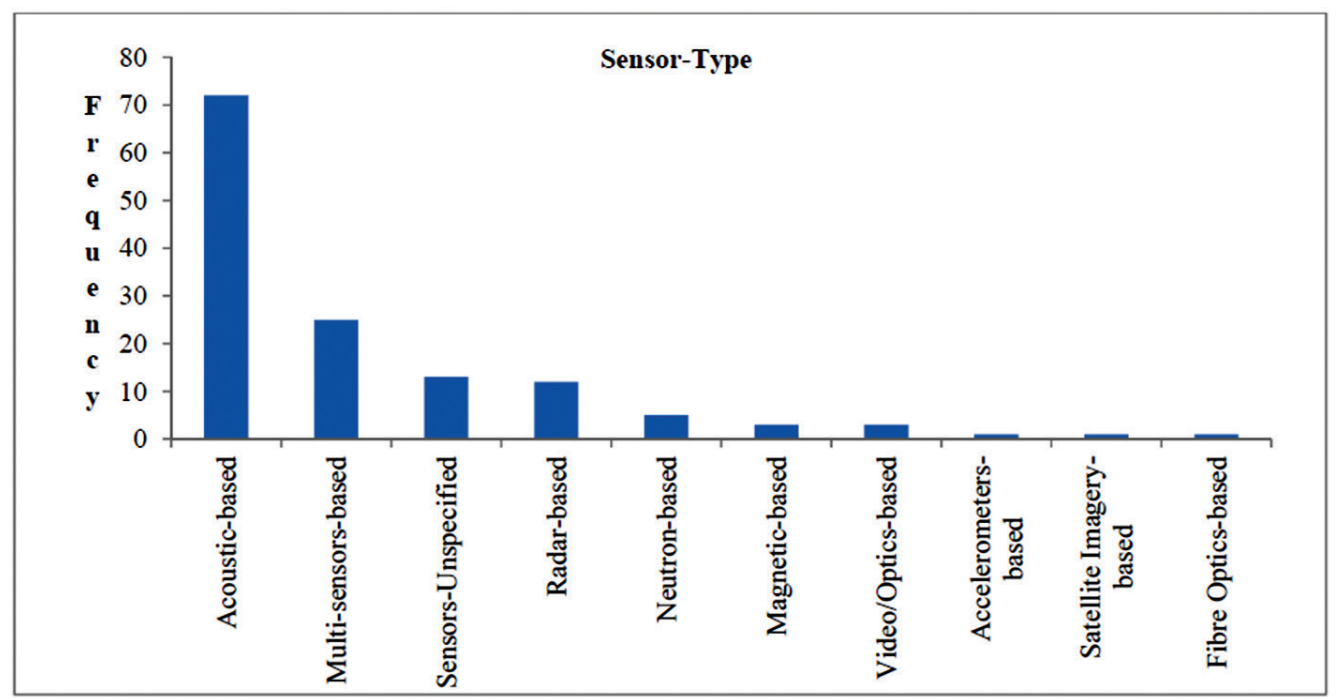

Figure 8.

Sensing technologies: Distribution of reviewed literature.

Of the 72 acoustic related papers, $26 \%$ are associated with active sensors such as sonars, $13 \%$ are associated with passive sensors such as hydrophones and sonobuoys, and $61 \%$ are non- specific about the type of acoustic sensors. Figure 9 shows this distribution. 


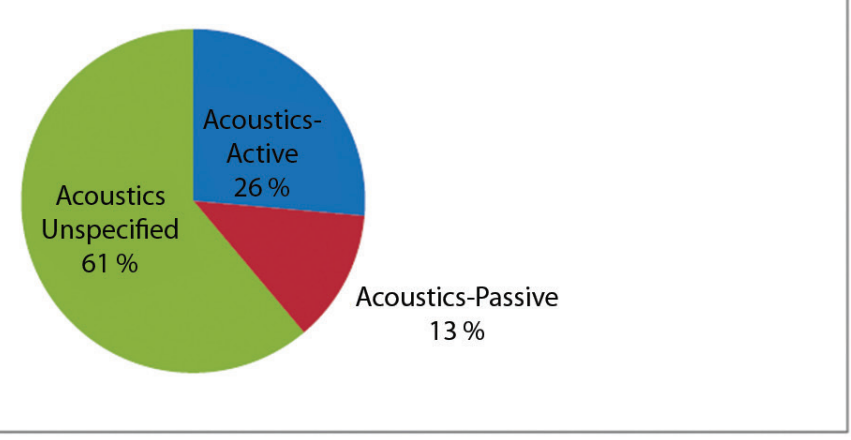

Figure 9.

Sensing technologies-Acoustics related: Distribution of reviewed literature.

It should be noted that for papers related to the deployment of autonomous underwater vehicles, AUVs, we identify the actual sensors used by the underwater vehicles (which are most often multiple sensors, combining two or more sensors), and in cases where the sensor types are not explicit, we include them under the "Unspecified sensor" category.

\section{DISCUSSIONS AND CONCLUSION}

In this paper, a review of recent works in underwater threat detection is presented. Classifications are shown based on authors' regional affiliations, solution approach/ methodologies, and research contributions. In addition, due to the nature of the review, a classification based on the type of sensing technology involved is also included.

Amongst others, Sensor placement and deployment within underwater/maritime framework is identified as an area requiring researchers' contributions. Compared to terrestrial sensor placement and deployment, underwater/marine sensor placement and deployment has not been widely studied in literature.

Although the authors recognize participation in crossregional collaborations among regions already active in the research area (especially between Europe and USA/Canada regions), the authors' opinion is that the area can benefit more from international collaborations, especially between these active regions and countries with vibrant maritime activities such as Qatar, Kuwait, Saudi Arabia, etc. in the Middle East region, and Nigeria, Guinea, Somalia, etc. in the Africa region. These two regions, though highly prone to the existing threats of terrorism and piracy have little or no visibility in the literature distribution.

Although this literature survey indicates the preponderance of acoustic sensors in comparison to other sensing technologies, it should be noted that practical implementations of maritime surveillance systems as seen in literatures dedicated to the development, deployment and analysis of these systems indicates that rather than the use of a specific sensing technology, the combination of various technologies with their unique benefits is the reality in most practical surveillance systems.

While acknowledging that the literature surveyed may not be a complete list of bibliography in the research area, the authors earnestly believe the results give an accurate representation of research trends in the period under review.

\section{ACKNOWLEDGEMENTS}

This paper was made possible by a National Priorities Research Program grant from the Qatar National Research Fund (a member of The Qatar Foundation). The statements made herein are solely the responsibility of the authors.

\section{APPENDIX:}

Supplemental Material: available on-line.

\section{REFERENCES}

Altay, N. and Green III, W. G., (2006), OR/MS research in disaster operations management, European Journal of Operational Research, 175(1), pp. 475-493., http://dx.doi.org/10.1016/j.ejor.2005.05.016

Akkaya, K. and Newell, A., (2009), Self-deployment of sensors for maximized coverage in underwater acoustic sensor networks, Computer Communications, 32(7), pp. 1233-1244.,

http://dx.doi.org/10.1016/j.comcom.2009.04.002

Galindo, G., and Batta, R., (2013), Review of recent developments in OR/MS research in disaster operations management, European Journal of Operational Research, 230(2), pp. 201-211.

http://dx.doi.org/10.1016/j.ejor.2013.01.039

Ghafoori, A. and Altiok, T., (2012), A mixed integer programming framework for sonar placement to mitigate maritime security risk, Journal of Transportation Security, 5(4), pp. 253-276.,

http://dx.doi.org/10.1007/s12198-012-0095-6 
Heidemann, J., Stojanovic, M. and Zorzi, M., (2012), Underwater sensor networks: applications, advances and challenges, Philosophical Transactions of the Royal Society A: Mathematical, Physical and Engineering Sciences, 370(1958), pp. 158175.,

http://dx.doi.org/10.1098/rsta.2011.0214

Heidemann, J., Ye, W., Wills, J., Syed, A. and Li, Y., (2006), Research challenges and applications for underwater sensor networking, Wireless Communications and Networking Conference, Las Vegas, USA, April 3-6,vol. 1, pp. 228-235. http://dx.doi.org/10.1109/WCNC.2006.1683469

Ibrahim, S., Al-Bzoor, M., Liu, J., Ammar, R., Rajasekaran, S. and Cui, J. H., (2013), General optimization framework for surface gateway deployment problem in underwater sensor networks, EURASIP Journal on Wireless Communications and Networking, 2013(1), pp. 1-13.,

http://dx.doi.org/10.1186/1687-1499-2013-128

IMO, (2012), International Shipping Facts and Figures - Information Resources on Trade, Safety, Security, Environment, available at: http://www.imo.org/KnowledgeCentre/ShipsAndShippingFactsAndFigures/ TheRoleandlmportanceofInternationalShipping/Documents/International\%20 Shipping\%20-\%20Facts\%20and\%20Figures.pdf, [accessed August 2014.].

Li, S., Meng, Q. and Qu, X., (2012), An overview of maritime waterway quantitative risk assessment models, Risk Analysis, 32(3), pp. 496-512., http://dx.doi.org/10.1111/j.1539-6924.2011.01697.x

Molyboha, A. and Zabarankin, M., (2012), Stochastic optimization of sensor placement for diver detection, Operations research, 60(2), pp. 292-312., http://dx.doi.org/10.1287/opre.1110.1032
Natarajarathinam, M., Capar, I. and Narayanan, A., (2009), Managing supply chains in times of crisis: a review of literature and insights, International Journal of Physical Distribution and Logistics Management, 39 (7), pp. 535-573.,

http://dx.doi.org/10.1109/WCNC.2006.1683469

Ngatchou, P. N., Fox, W. L. and El-Sharkawi, M. A., (2006), Multiobjective multistatic sonar sensor placement, Proc. IEEE Congress on Evolutionary Computation, Vancouver, Canada, July 16-21, pp. 2713-2719., http://dx.doi.org/10.1109/CEC.2006.1688648

OECD, (2003), Security in Maritime Transport: Risk factors and Economic Impact. Maritime Transport Committee, Directorate for Science, Technology and Industry, available at: http://www.oecd.org/sti/transport/maritimetransport/18521672.pdf, [accessed August 2014.].

Stolkin, R. and Florescu, I., (2007), Probabilistic analysis of a passive acoustic diver detection system for optimal sensor placement and extensions to localization and tracking, Proc. Oceans 2007,Vancouver, Canada, September 29, pp. 1-6., http://dx.doi.org/10.1109/OCEANS.2007.4449203

United States Department of Transportation Maritime Administration, (2007), The Maritime Administration and the U.S. Marine Transportation System: A Vision for the 21st Century, available at:

http://www.marad.dot.gov/documents/Vision_of_the_21st_Century_10-29.pdf, [accessed August 2014.]. 